\title{
Development of a Direct Trypan Blue Exclusion Method to Detect Cell Viability of Adherent Cells into ELISA Plates
}

\author{
Selcen Çelik Uzuner \\ Department of Molecular Biology and Genetics, Faculty of Science, Karadeniz Technical University, Trabzon, \\ 61080, TURKEY \\ selcen.celik@ktu.edu.tr
}

Received: 28 December 2017

Accepted: 8 February 2018

DOI: $10.18466 /$ cbayarfbe. 372192

\begin{abstract}
Cell viability detection is important in cell culture applications including measurement of cell proliferation i.e for understanding cytotoxic effects of compounds on cells. There are some cell viability methods based on fluorescence or non-fluorescence detection. More simplified evaluation for cell viability, such as trypan blue staining, can be preferred before performing fluorescence assays. This appears advantageous when to have a large number of cell samples in ELISA plates after treatments with different concentrations of drug candidates. Thus, further fluorescence assays can include less concentrations rather than experiencing all used along 96well plates. For this, trypan blue exclusion method is an option. Traditionally, treated cells are harvested by centrifugation and incubated with trypan blue within tubes followed by transferring the mixture into a hemacytometer with two chambers and assessed under the microscope. Nevertheless, using a hemacytometer limits practicability of this method when analyzing various cell samples into 96-well plates at the same time. This study was aimed to adapt trypan blue method to in situ staining of adherent cells cultured on ELISA plates. For this, cells were fixed with different fixatives after trypan blue incubation to maintain cells in impenetrable meshwork, and paraformaldehyde was the most effective fixative. This modified protocol was validated by testing the effect of dimethylsulfoxide-a cytotoxic agent-on cells, and expectedly found that cell viability reduced with higher concentrations of dimethylsulfoxide suggesting that in situ detection of cell viability by trypan blue can be a useful tool for preliminary detection of cells cultured on ELISA plates before performing automatized experiments with such flow cytometer and/or microplate reader.
\end{abstract}

Key words: Cell viability, cell death, adherent cells, trypan blue exclusion, ELISA plate, fixation

\section{Introduction}

Cell viability assessment is one of the basic methods to figure out the wellness of cells in culture. This assessment has been also commonly used in a wide range of biochemistry studies including evaluation of the effects of new drug candidates on relevant cells. There are a number of methods for the detection of cell viability such as fluorescence- or non-fluorescence based methods. Many of these methods require expensive commercial kits, fluorescence dyes/antibodies and instruments such as flow cytometry, fluorescence microscope or ELISA-plate reader. Although trypan blue exclusion method is considered as a subjective method to some degree, it is the cheapest and the common method for understanding the ratio of cell viability in particular for gathering preliminary data by traditional light microscopes. However, one of the disadvantages of trypan blue exclusion method is that it cannot allow a large number of treatments to be assessed in a limited time, i.e. if cells cultured in 96-wells, because standard method for trypan blue staining is performed using a hemacytometer with two chambers only. It means that a hemacytometer can be used to detect cell viability of differentially treated two cells only at once. Therefore, the use of hemacytometer is not applicable and reasonable while experiencing many cells concurrently. The other reason is that it may lead to false positive staining since live cells can undergo cell death while waiting in tubes or onto hemacytometer to be counted. In this study, it was therefore aimed to modify trypan blue protocol for detection of cell viability of cells cultured in ELISA plates, namely in situ staining of cells into 96well plates, by the assessment of conventional light microscope. This modified method (i) simplifies simultaneous staining of cells with no necessity for detaching cells from culture surface and using a hemacytometer and (ii) minimizes false positive staining during evaluation of many samples. This protocol was also validated using cells treated with dimethylsulfoxide and showed that dimethylsulfoxide at more than $0.5 \%$ final concentration reduced cell viability rate compared to ethanol-treated cells. 


\section{Materials and Methods}

\subsection{Cell culture}

Cells used were mouse embryonic fibroblasts at first passage, and cultured in complete media containing DMEM (WISENT Inc., Québec, Canada, Cat\# 319-007CL), $10 \%$ (v/v) fetal bovine serum (Capricorn Scientific GmbH, Ebsdorfergrund, Germany, Cat\# FBS-11A), 1\% (v/v) non-essential amino acids (Capricorn, Cat\# NEAAB) and $1 \%(\mathrm{v} / \mathrm{v})$ penicillin-streptomycin (Gibco, Carlsbad, CA, USA, Cat\# 15140-122). Complete media was filtered by a 0.22 micron filter (Aisimo Corp. Co, London, England), and warmed at $37^{\circ} \mathrm{C}$ before use. Cells were seeded on a 96-well plate as around 5000 cells per well, and incubated at $37^{\circ} \mathrm{C}$ in a humidified incubator with $5 \% \mathrm{CO}_{2}$.

\subsection{Solvent exposure of cells}

Cells were treated either with (i) dimethylsulfoxide (DMSO) (Sigma-Aldrich, St Louis, USA, D2650) or (ii) absolute ethanol (Sigma, Cat\# 32221) for 24h, 48h or $72 \mathrm{~h}$ at the concentrations of $0.1 \%(\mathrm{v} / \mathrm{v}), 0.25 \%(\mathrm{v} / \mathrm{v}), 0.5 \%$ $(\mathrm{v} / \mathrm{v}), 1 \%(\mathrm{v} / \mathrm{v}), 1.5 \%(\mathrm{v} / \mathrm{v})$ or $2 \%(\mathrm{v} / \mathrm{v})$ in complete media. Control cells were untreated. Cells were then stained with trypan blue and fixed with 4\% paraformaldehyde (PFA) followed by microscopy assessment as detailed below.

\subsection{Trypan blue staining}

Cells were incubated with $0.4 \%$ trypan blue solution (Sigma, Cat\# T4049) followed by fixation step. For this staining, confluent cells were washed with PBS (phosphate buffered saline) (PAN-Biotech, Aidenbach, Germany, Cat\# P04-36500) for three times, then incubated with trypan blue either at final concentrations $0.4 \%, 0.2 \%(\mathrm{v} / \mathrm{v})$ or $0.1 \%(\mathrm{v} / \mathrm{v})\left(\right.$ in $\left.\mathrm{dH}_{2} \mathrm{O}\right)$ for 10 minutes at room temperature (RT). Cells were washed with PBS again after trypan blue treatment.

\subsection{Fixatives}

After trypan blue treatment, cells were fixed for half an hour at RT. Fixatives used were glutaraldehyde (Merck, Darmstadt, Germany, Cat\# 820603), formaldehyde (Merck, Cat\# 104003), methanol (Merck, Cat\# 106009) and PFA (ChemSolute ${ }^{\circledR}$, Renningen, Germany, Cat\# 8416-0500). Original concentrations were 25\%, 37\%, $99 \%$ for glutaraldehyde, formaldehyde and methanol, respectively. Glutaraldehyde, formaldehyde and methanol were diluted to lower concentrations with sterile $\mathrm{dH}_{2} \mathrm{O}$. For $4 \%$ PFA solution, PFA $(0.4 \mathrm{~g})$ was dissolved within $\mathrm{ddH}_{2} \mathrm{O}(10 \mathrm{ml})$ and $1 \mathrm{M} \mathrm{NaOH}(15 \mu \mathrm{l})$ was added into this. This mix was stirred at $37^{\circ} \mathrm{C}$ for 30 minutes until PFA powder became clear. PBS (0.096 g) was added after PFA dissolved completely, and $\mathrm{pH}$ was adjusted to 7.4. All fixatives were prepared fresh for each use. After fixation, cells were washed with 1xPBS until fixatives removed completely.

\subsection{Microscope assessment}

Stained and fixed cells kept in 1xPBS were observed by 20x objective of AxioVert.A1 inverted microscope (Carl Zeiss, Germany). The representative photos for each well were captured from different areas of a well using the camera AxioCam ICc 1 (Zeiss). Live (unstained with trypan blue) and dead (stained with trypan blue) cells in these photos were counted for each treatment. Count tool of Adobe Photoshop software was used for this. Experiments were performed as three independent replicates.

\subsection{Statistical analysis}

Statistical analyses were performed using SPSS software (version 23), and numbers of live and dead cells were compared by UNIANOVA test. The differences between two groups were analyzed by Post-Hoc test. $\mathrm{P}$ values less than 0.05 were considered as significant. Bar graphs show error bars for $+/$ - standard of the mean.

\section{Results and Discussion}

The most important issue at the first step was that washing with PBS (without calcium and magnesium) detached cells from plate wells. To prevent this, cells were washed with PBS containing calcium and magnesium maintained cells attached on surface. Calcium and magnesium are important divalent cations since these ions maintain proteins functioning in cell adhesion to the surface $[1,2]$. In general, these cations are advised to be included in washing buffers unless processing to detach cells by trypsin digestion for subculture. On the contrary, after fixation, cells were washed again with PBS (without calcium and magnesium) and no cell detachment was observed due to that fixatives already stabilized cell metabolism as its in native state [3]. Alternatively, cells can be washed with complete media or DMEM solution only since they already include these cations. After washing, cells were treated with trypan blue staining as indicated above. For a long time, trypan blue dye has been generally used at $0.2 \%$ concentration in independent studies on different cell types [4-7]. The dye penetrates into membranes of dead cells since dead cells have damaged membranes, whereas the dye is excluded from live cells with intact cell membrane so that dead cells are seen as blue under light microscope (Figure 4a) [8]. There was no significant difference in the detection of cell viability between trypan blue concentrations (data not shown) so that further experiments were performed with $0.2 \%$ trypan blue as stated in previous studies [4-7]. Following trypan blue (TB), cells were treated with different fixatives: glutaraldehyde (Glu), formaldehyde (Form), methanol (Met) or paraformaldehyde (PFA) at different concentrations for half an hour at room temperature, and control cells were not treated with any fixative. Variations in the detected percentages of viable cells were found after different fixatives; (Figure 1). Expectedly, cells with no fixative had the most fluctuated ratio (represented by a high value of standard error) 
suggesting the necessity of fixation step in the protocol, and viability of cells (\%) after fixatives, except PFAtreated cells, was not significantly different than viability of unfixed cells since the viability percentages fell into the same range with cells without fixative. But, PFA fixation differently resulted in the highest viability $(p<0.05)$. Only PFA-fixed cells showed significant difference in the detection of viable cells compared to other fixatives regardless of their concentrations (Figure 1). Figure 2 shows some representative images of microscopy observation. Examples of dead cells stained as blue are indicated with red arrows. In general, fibroblast cell morphology was remained after fixation (Figure 2a, 2c), but 99\% methanol fixation resulted in round-shaped cells indicating that $99 \%$ methanol fixation did not stabilize cells well within fibroblast structure and $99 \%$ methanol also evaporated until assessment so that this fixative is not suitable (Figure 2b). Figure 3 summarizes the workflow of the modified protocol.

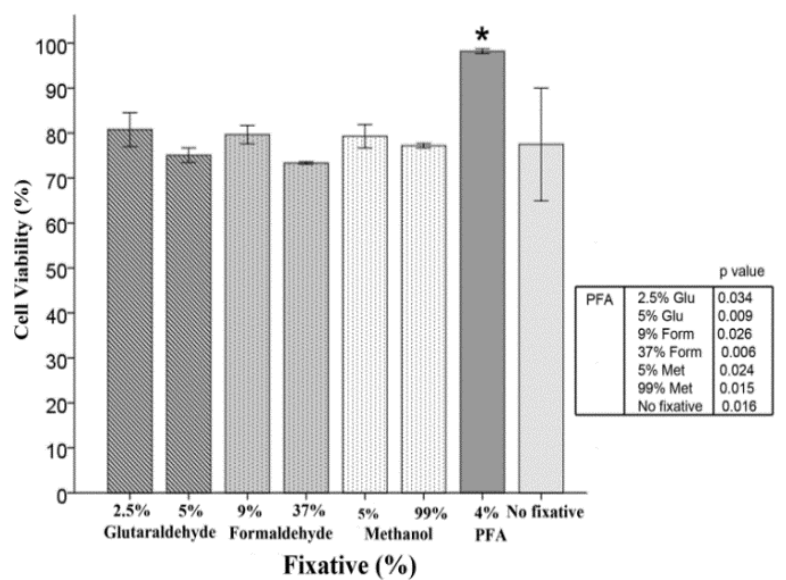

Figure 1. Cell viability (\%) detected using trypan blue exclusion followed by fixatives.. $P$ values for comparisons of each treatment with PFA are given in the box. All $\mathrm{p}$ values less than 0.05 are considered as significant. Error bars show $+/$ - standard error of the mean.

As a result, PFA (4\%) fixation after $0.2 \%$ trypan blue staining was included in this modified protocol. Paraformaldehyde is the polymerized form of formaldehyde and the most effective fixative commonly used in staining protocols [9-13]. Because PFA does not only produce cross-links between proteins but can also fasten proteins into impenetrable network of cellular components. Another reason is that it causes the minimum false positive staining [14]. Aldehyde groups are advantageous fixatives since they are able to stabilize cellular components and preserve protein structures by cross-links between such cellular proteins so that this allows prolonged and comprehensive microscope observations [15]. However, glutaraldehyde is not as effective as formaldehyde to induce cross-links between proteins and DNA, but within proteins [3]. One of the important disadvantages of fixation with alcohol derivatives is that they can cause protein denaturation during fixation process [15].

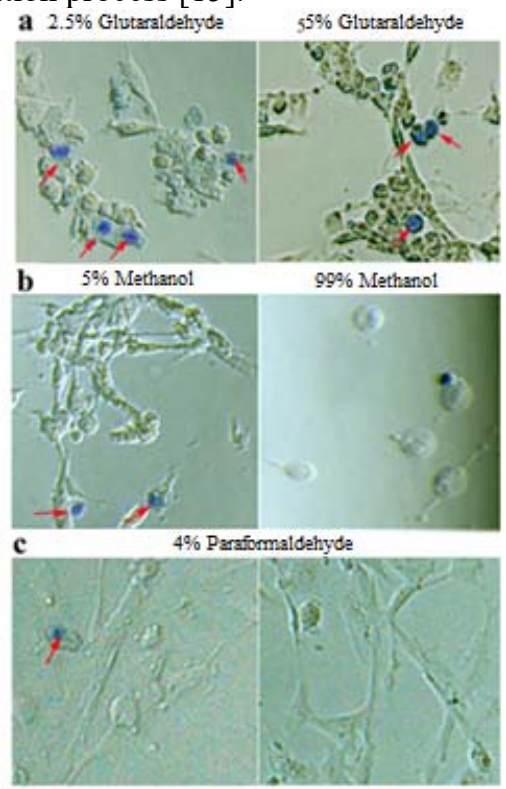

Figure 2. Representative microscope photos for live and dead cells. Examples of dead cells after (A) glutaraldehyde, (B) methanol and (C) paraformaldehyde fixation are indicated with red arrows. Round-shaped cells are seen after $99 \%$ methanol fixation. Photos were taken using a 20x objective.

Workflow for direct method to detect cell viability in ELISA plates Cell culture ( $\sim 5000$ cells per well $)$

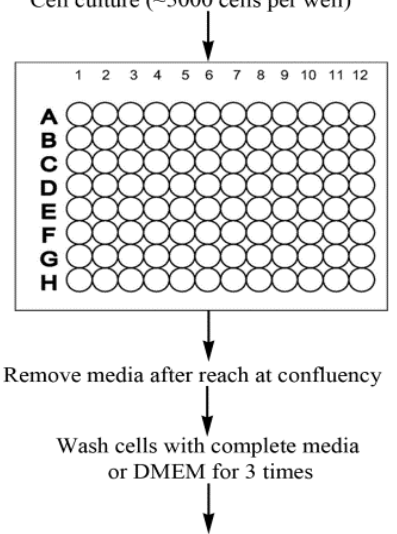

Add $50-100 \mu 10.2 \%$ trypan blue (TB) solution (in sterile $\mathrm{dH} 2 \mathrm{O}$ ) to each well and incubate for $10 \mathrm{~min}$ at room temperature (RT)

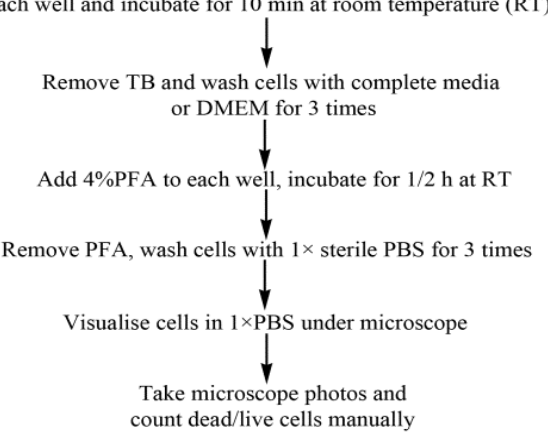

Figure 3. Workflow for optimized method to directly detect cell viability of cells cultured into ELISA plate wells. 
There is a study that described trypan blue staining of cells cultured on coverslips and included coverslip mounting after PFA fixation [10]. Although this study was also performed with PFA fixation, this coverslip protocol does not appear useful for studies that include too many treatments/samples in 96-well plate cultures.

The applicability of this protocol for cell viability was then assessed after treatment with a known cytotoxic agent, DMSO, is used. Ethanol was used as a control agent. Control cells were untreated. Higher concentrations of ethanol did not significantly reduce cell viability, and the differences between $24 \mathrm{~h}, 48 \mathrm{~h}$ and $72 \mathrm{~h}$ after ethanol were similar in each concentration (Figure $4 a)$. The trend for each time point was also similar in untreated cells. On the other hand, DMSO at 1-2\% concentration induced increased cell death, however the viability detected less than $1 \%$ was quite corresponded to cells treated with ethanol less than $1 \%$ (Figure $4 b$ ). Figure 5 shows some representative microscope images of cells treated with either DMSO or ethanol. It can suggest that DMSO up to $0.5 \%$ is not toxic to fibroblast cells whereas ethanol appears safe in the all concentrations experienced compared to untreated cells. DMSO and ethanol are widely used solvents for preparation of such drug candidate organic compounds [16, 17]. DMSO is also used as a chemotherapeutic agent to induce death of cancer cells [18]. On the other hand, DMSO is able to have cytotoxic effects on normal cells as many of chemotherapeutics [19]. The toxicity of DMSO has been studied in a range of cells. For instance, $0.1 \%$ and $0.5 \%$ DMSO concentration did not have a significant toxicity on macrophages and epithelial cells as well as on breast cancer cells [20].


Figure 4. Cell viability (\%) detected after treatment of solvents with fibroblasts. Cells were treated with either ethanol or DMSO at $0.1-2 \%$ concentrations for $24 \mathrm{~h}, 48 \mathrm{~h}$ or $72 \mathrm{~h}$. Control cells were untreated $(0 \%)$. Cell viability rates after ethanol treatments are similar to untreated cells, however cells treated with DMSO $(\geq 1 \%)$ show decreased cell viability. Error bars show $+/$ - standard error of the mean.

A higher concentration (1\%) of DMSO did not have an effect on death of nervous system cells such astrocyte whereas $5 \%$ of it damaged mitochondrial integrity of cells and caused cell death [21]. Although ethanol is more harmless than DMSO, acute exposure of cells with 30$40 \%$ ethanol resulted in death of all of normal and cancer cells exposed [22]. Cell viability assay modified in this study showed that DMSO at more than $1 \%$ concentration resulted in significant cell death; however ethanol-treated cells at counterpart concentrations did not undergo remarkable cell death. 


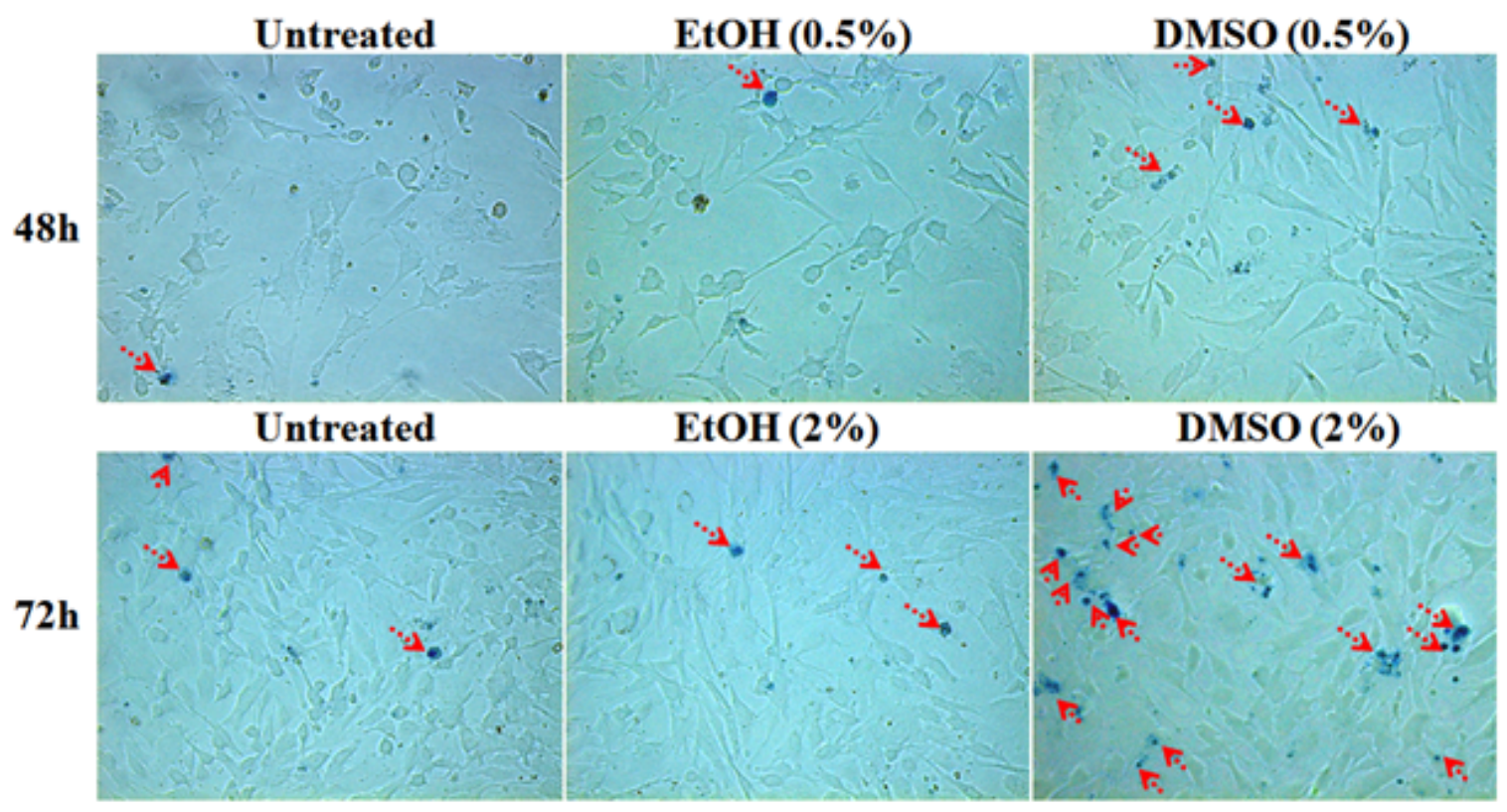

Figure 5. Representative microscope images of live and dead cells after solvent treatments. Cells were treated with either ethanol of DMSO as stated in Figure 4. Dead cells (blue) are indicated with red arrows. Cell viability decreased when cells treated with DMSO at $2 \%$. However ethanol did not induce a different viability profile than untreated cells. Photos were taken using a 20x objective.

\section{Conclusions}

The modified protocol in this study is the first protocol for in situ trypan blue staining of cells attached into ELISA plate wells. This protocol briefly includes PFA fixation of trypan blue stained cells. However, counting process via trypan blue exclusion method is highly subjective. Therefore, the present work suggests using of this protocol for preliminary evaluation of effect of drug candidate compounds on relevant cell lines. Thus, researchers can select the most effective concentrations via this method and go through the more detailed assays for cell viability using such fluorescence-based tools and other molecular analyses such genome-based tools.

A limitation of this modified method is to loss a degree of dead cells while washing the wells at the first step of the protocol. This may cause false negative staining. To avoid this, the media including floating dead cells can be incubated with trypan blue after centrifugation and the number of counted dead cells by classical hemacytometer can be also included in the analyses with cells counted via in situ staining. Nevertheless, it should be noted that this limitation is also the case for fluorescence-based assays of cell viability since all those kinds of assays include cell washing as a first step of protocol. In conclusion, traditional trypan blue exclusion method was modified to be used for simultaneous detection of viability of cells cultured into ELISA plate wells. This method is able to reduce the risk for false positive staining of trypan blue during prolonged periods of evaluation, and therefore facilitates the determination of cell viability by trypan blue exclusion method in a more reliable and straightforward way.

Acknowledgments: This work was supported by a grant (FHD-2016-5520) from the Scientific Research Projects Coordination Unit of Karadeniz Technical University. I thank Elif Ayazoglu Demir for assisting preparations and experiments.

Conflict of interest: Author declares no conflict of interest exists.

\section{References}

1. Takeichi, M. and T.S. Okada, Roles of Magnesium and CalciumIons in Cell-to-Substrate Adhesion. Experimental Cell Research, 1972. 74(1): p. 51-\&.

2. Day, E.S., L. Osborn, and A. Whitty, Effect of divalent cations on the affinity and selectivity of alpha 4 integrins towards the integrin ligands vascular cell adhesion molecule-1 and mucosal addressin cell adhesion molecule-1: Ca2+ activation of integrin alpha 4 beta 1 confers a distinct ligand specificity. Cell Communication and Adhesion, 2002. 9(4): p. 205-219.

3. Sewell, B.T., C. Bouloukos, and C. von Holt, Formaldehyde and glutaraldehyde in the fixation of chromatin for electron microscopy. Journal of Microscopy, 1984. 136(Pt 1): p. 103-112.

4. Tennant, J.R., Evaluation of the Trypan Blue Technique for Determination of Cell Viability. Transplantation, 1964. 2(6): p. 685-694. 
5. Park, S., et al., Protocol for Vital Dye Staining of Corneal Endothelial Cells. Cornea, 2012. 31(12): p. 1476-1479.

6. Mody, K.T., et al., Freeze-drying of ovalbumin loaded mesoporous silica nanoparticle vaccine formulation increases antigen stability under ambient conditions. International Journal of Pharmaceutics, 2014. 465(1-2): p. 325-332.

7. Okahashi, N., et al., Hydrogen Peroxide Contributes to the Epithelial Cell Death Induced by the Oral Mitis Group of Streptococci. Plos One, 2014. 9(1).

8. Strober, W., Trypan blue exclusion test of cell viability. Current Protocols in Immunology, 2001. Appendix 3: p. Appendix 3B.

9. Tanaka, K.A.K., et al., Membrane molecules mobile even after chemical fixation. Nature Methods, 2010. 7(11): p. 865-866.

10. Perry, S.W., L.G. Epstein, and H.A. Gelbard, In situ Trypan Blue staining of monolayer cell cultures for permanent fixation and mounting. Biotechniques, 1997. 22(6): p. 1020-\&.

11. Mihara, K., T. Nakayama, and H. Saitoh, A Convenient Technique to Fix Suspension Cells on a Coverslip for Microscopy. Current Protocols in Cell Biology, 2015. 68: p. 4 $301-10$.

12. Schnell, U., et al., Immunolabeling artifacts and the need for livecell imaging. Nature Methods, 2012. 9(2): p. 152-158.

13. Kading, R., M. Crabtree, and B. Miller, Inactivation of infectious virus and serological detection of virus antigen in Rift Valley fever virus-exposed mosquitoes fixed with paraformaldehyde. Journal of Virological Methods, 2013. 189(1): p. 184-188.

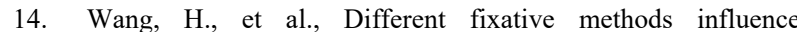
histological morphology and TUNEL staining in mouse testes. Reproductive Toxicology, 2016. 60: p. 53-61.
15. Thavarajah, R., et al., Chemical and physical basics of routine formaldehyde fixation. Journal of Oral and Maxillofacial Pathology, 2012. 16(3): p. 400-405.

16. Amer, S., N. El-Wakiel, and H. Ei-Ghamry, Synthesis, spectral, antitumor and antimicrobial studies on $\mathrm{Cu}(\mathrm{II})$ complexes of purine and triazole Schiff base derivatives. Journal of Molecular Structure, 2013. 1049: p. 326-335.

17. Bekircan, O., B. Kahveci, and M. Kucuk, Synthesis and anticancer evaluation of some new unsymmetrical 3,5-diaryl4H-1,2,4-triazole derivatives. Turkish Journal of Chemistry, 2006. 30(1): p. 29-40.

18. Da Violante, G., et al., Evaluation of the cytotoxicity effect of dimethyl sulfoxide (DMSO) on Caco2/TC7 colon tumor cell cultures. Biological and Pharmaceutical Bulletin, 2002. 25(12): p. $1600-163$.

19. Qi, W., D. Ding, and R.J. Salvi, Cytotoxic effects of dimethyl sulphoxide (DMSO) on cochlear organotypic cultures. Hear Research, 2008. 236(1-2): p. 52-60.

20. Jamalzadeh, L., et al., Cytotoxic Effects of Some Common Organic Solvents on MCF-7, RAW-264.7 and Human Umbilical Vein Endothelial Cells. The Avicenna Journal of Medical Biotechnology, 2016. 4(1): p. 10-33453.

21. Yuan, C., et al., Dimethyl Sulfoxide Damages Mitochondrial Integrity and Membrane Potential in Cultured Astrocytes. Plos One, 2014. 9(9).

22. Tapani, E., et al., Toxicity of ethanol in low concentrations Experimental evaluation in cell culture. Acta Radiologica, 1996. 37(6): p. 923-926. 